\title{
Study on the Lifting Force of Control Valve in the Process of Opening
}

\author{
Xiaozeng Xie ${ }^{1, a}$, Yun Zhang ${ }^{2, b}$, Shushuai Feng ${ }^{3, c}$, Xin Liu ${ }^{4, d}$ \\ Shanghai Marine Equipment Research Institute \\ Shanghai 200031 China \\ axiexiaozeng@hrbeu.edu.cn,

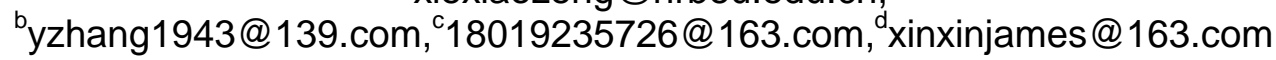

Keywords: The G-I type control valve; lifting force; numerical simulation; theory analysis

Abstract. The control valve is widely used in the steam turbine, rely mainly on the motion of the valve stem changes the relative position of other t-rims, to achieve the purpose of regulating the steam quantity. Currently the control valve in service often occur the fault as a result of the valve is difficult to open. The G-I type control valve is taken as the research object in this paper, the lifting force is studied in the process of opening using the method of theory analysis combined with the numerical simulation.

\section{Introduction}

Steam turbine start-stop and change its power cannot be separated from the control valve's control, and the control of control valve is mainly determined by the stem lifting force. In the design of the control valve, usually set the spring compression initial displacement, forming the spring force exerted on the valve stem, to the control valve in the closed state. If you would like to open the valve, the hydraulic oil motive to stem a upward force, to make the valve stem and valve disc to move up, the flow area of valve plate and valve seat formed gradually from zero to the maximum, which implements the process of control valve from closed to fully open.

If the initial spring force is too small, it may cause the sealing pressure on the valve disc and valve seat contact surface is not enough when the control valve in the closed, and resulting in leakage phenomenon; If more than the oil motive for lifting force, may cause the valve can't open, even can not into the steam, seriously affecting the performance of the steam turbine units, and even cause accidents. Therefore, in the design process of the control valve, the steps of calculating stem lifting force integral, at the same time, but also an important basis for the selection of the valve.

\section{The theoretical analysis of the lifting force in the pre process}

For G-I type of control valve, the structure of pre opening valve as shown in figure 1:

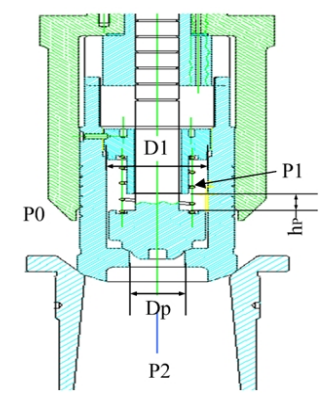

Fig.1 Pre opening valve structure

In Figure 1, the valve is in the open state at the beginning, P0 for the steam inlet pressure, P1 for balance cavity pressure on the pre opening valve, P2 for the steam outlet pressure, D1 for the balance cavity diameter, hp for the pre opening valve stroke, Dp for the pre opening valve contact diameter, in addition to the valve disc cutting hole is rectangular, $\mathrm{S}$ for the cross sectional area. 
The steam flow into the balance cavity of the pre opening valve through four tangential holes on the valve disc when the control valve is closed, form the balance chamber pressure P1, along with the pre opening valve open, the steam in the balance cavity flow into the back of valve, attaining the aim of reducing pressure difference before and after the valve. Appeared the twice throttle in the process, the first time for the steam by tangential hole into the balance cavity, $S_{1}=4 S$ for the throttling area, the second time for the steam in balance cavity flow into the back of valve, $S_{2}=\pi D_{p} h_{p}$ for the throttling area.

Two throttle the flow formula as follows:

$$
\begin{aligned}
& Q_{1}=\mu_{1} \cdot S_{1} \sqrt{\frac{2 g}{r}\left(P_{0}-P_{1}\right)} \\
& Q_{2}=\mu_{2} \cdot S_{2} \sqrt{\frac{2 g}{r}\left(P_{1}-P_{2}\right)}
\end{aligned}
$$

According to the laws of the flow conservation $Q_{1}=Q_{2}$, the formula (1) can be obtained.

$$
\begin{aligned}
P_{1}-P_{2} & =\frac{S_{1}^{2}}{S_{1}^{2}+S_{2}^{2}}\left(P_{0}-P_{2}\right) \\
& =\frac{1}{1+\alpha \cdot h_{p}^{2}}\left(P_{0}-P_{2}\right)
\end{aligned}
$$

Where, $\alpha=\left(\frac{\pi D_{P}}{4 S}\right)^{2}$.

$\alpha$ can be seen as the structure parameters of control valve, only have a connection with contact diameter of the pre opening valve and size of tangential hole on the valve disc. No matter in the design or selection of control valve in the given application, the parameter is constant.

The formula (2) can be obtained using the analysis of the force on the valve stem.

$$
F=G+F_{s}+F_{k}=G+\frac{\pi}{4} D_{1}^{2}\left(P_{1}-P_{2}\right)+F_{c}+k \cdot h_{p}
$$

Where, $F$ is the sum of valve stem by force in addition to acting force of the hydraulic oil motive; $G$ is the gravity of valve stem; $F_{\mathrm{s}}$ is the acting force of steam; $F_{\mathrm{k}}$ is the acting force of spring; $F_{\mathrm{c}}$ is the initial acting force of spring; $k$ is the spring stiffness coefficient.

The formula (3) is obtained by the formula (1) and formula (2).

$$
F=\mathrm{A}+\frac{\mathrm{B}\left(P_{0}-P_{2}\right)}{1+\alpha \cdot h_{p}^{2}}+k \cdot h_{p}
$$

Where, $A=G+F_{c}, B=\pi D_{1}^{2} / 4$ is the contact area of pre opening valve.

For the design of the given control valve, $\mathrm{A}$ and $\mathrm{B}$ is a constant. The lifting force of control valve in the process of opening mainly depends on the stroke of the pre opening valve, structural parameters of the control valve and the pressure difference between the before and after the valve, the relationship of the lifting force and pre stroke of the stem as shown in figure 2. 


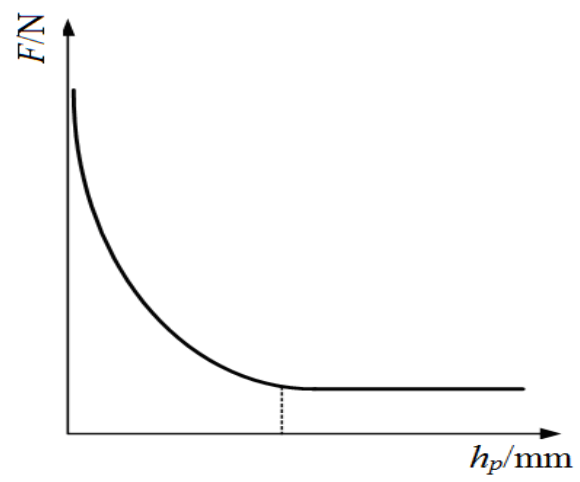

Fig. 2 The relationship curve of F-hp

The relationship curve of F-hp shows the conclusion of increasing pre opening valve stroke can reduce the valve stem lifting force. The valve lifting force decreases rapidly with the increase of the pre opening stroke, the lifting force to reduce speed gradually slow down until it leveled off when reaches a certain value. So the behavior of reducing pre opening valve stroke is not economic in order to reduce valve lifting force. At the same time, the steam turbine in limited space, reserved for the hydraulic oil motor starting space is limited, it may cause the case of the valve can not fully open if the pre opening valve stroke is too large.

\section{The numerical simulation analysis of lifting force in the process of opening}

The model of pre opening valve of control valve was reestablished,the pre-opening stroke of the pre-opening valve is set to $4.75 \mathrm{~mm}$. Because here only to explore the relationship of the pre-stroke and lift, so the control valve is set to off. To select the pre-stop valve of the typical trip (minimum $0.5 \mathrm{~mm}, 0.25 \mathrm{~mm}$ increments, the maximum $4.75 \mathrm{~mm}$ ), the pre-lift valve lift force is obtained using the numerical simulation method. When the pre-valve lift is $3 \mathrm{~mm}$, the flow field distribution is as follows:

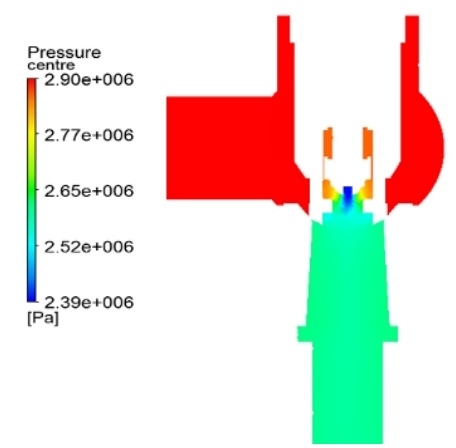

(a) Pressure filed distribution

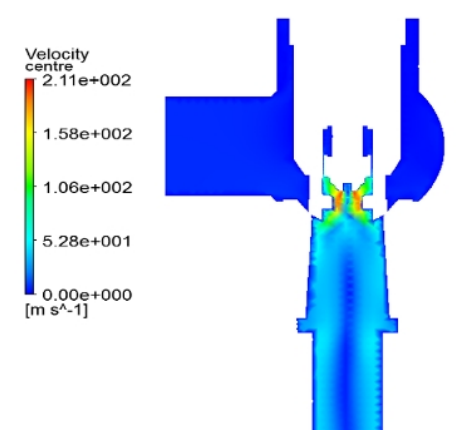

(b) Velocity filed distribution

Fig. 3 The valve flow field in pre-opening process

Figure 3 can be seen, the inlet valve and the outlet pressure distribution is more uniform, changes in the main pre-valve, the pre-opening valve pressure decreases, and because the reasons for throttling, changes more turbulent; And the speed is just the opposite, in the inlet and outlet evenly distributed in the pre-opening valve increases and disorder distribution.

Since FLUENT has a dimensionless Newtonian (N) option in the monitoring window, the lift is replaced by the lift factor, which is defined as:

$$
C_{d}=\frac{F}{\frac{1}{2} \rho u^{2} L}
$$

In Fluent, the density, the velocity, and characteristic length of the reference values can be defined as 2,1 and 1, respectively, and the denominator in equation (4) is numerically equal to lift. 
Through the numerical simulation of the typical opening flow field, the lift coefficients corresponding to different pre-stroke are shown in table 1.

Table 1 The lift coefficient under different the pre-opening stroke

\begin{tabular}{c|c|c|c|c|c|c}
\hline Stroke/mm & 0.5 & 0.75 & 1 & 1.25 & 1.5 & 1.75 \\
\hline$i=0.585$ & 3635.16 & 3582.88 & 3532.08 & 3494.53 & 3470.6 & 3468.76 \\
\hline$i=0.9$ & 11157.9 & 10852.48 & 10692.26 & 10604.64 & 10483.22 & 10429.4 \\
\hline \hline Stroke/mm & 2 & 2.25 & 2.5 & 2.75 & 3 & 3.25 \\
\hline$i=0.585$ & 3439.67 & 3431.57 & 3430.84 & 3428.63 & 3424.58 & 3424.21 \\
\hline$i=0.9$ & 10415.63 & 10354.29 & 10335.52 & 10325.5 & 10319.25 & 10317.99 \\
\hline \hline Stroke/mm & 3.5 & 3.75 & 4 & 4.25 & 4.5 & 4.75 \\
\hline$i=0.585$ & 3424.21 & 3423.84 & 3423.47 & 3428.26 & 3422 & 3422.74 \\
\hline$i=0.9$ & 10315.49 & 10314.24 & 10300.47 & 10312.99 & 10311.74 & 10325.5 \\
\hline
\end{tabular}

In the field test, the lifting force coefficient is usually used to measure the lift force of the valve. The coefficient of interaction and the theory of the valve by the steam power ratio, the control valve of the theory of stress were $3682 \mathrm{~N}$ (pressure ratio 0.585 ) and $12517 \mathrm{~N}$ (pressure ratio 0.9), the relationship between different pre starting stroke and lifting force as shown in figure 4 .

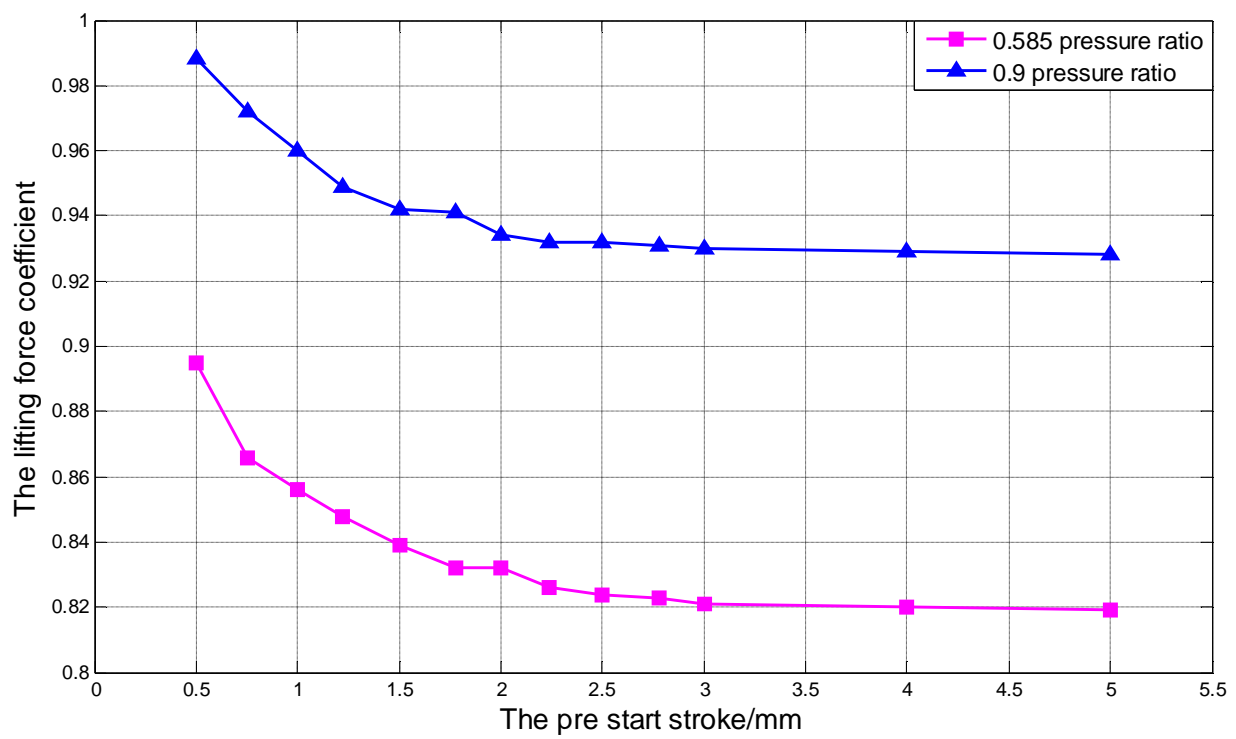

Fig.4 The curve of relationship between pre-stroke and lift coefficient

Figure 4 shows that the stem lifting force along with the increase of the pre start stroke gradually decreases, when the pre start stroke reached, the lifting force changes tend to be gentle. In the design of the valve, the steam turbine within the limited space and consider the economic and other factors, the valve opening and pre trip should be taken as $3 \mathrm{~mm}$ is appropriate, not only to meet the requirements of reducing lifting force, and not too occupied space in steam turbine.

\section{Numerical Simulation Analysis of Lifting Force in Regulating Process}

When the valve before the start of the process, the stem began to control the displacement of the valve disc to achieve the work requirements of different circulation capacity, this process is also known as the regulation process. In this scheduling process, the size of the lifting force is closely related to the position of the valve disc. Because lift valve disc position can be used to measure the stem.

Numerical simulation of the flow field in ten typical position open degree of the valve stem is carried out to monitor the lift coefficient of the valve disc and the valve stem. The numerical value is shown in table 2. 
Table 2 The lift coefficient under different open degrees

\begin{tabular}{c|c|c|c|c|c}
\hline Stroke/mm & 6 & 12 & 18 & 24 & 30 \\
\hline$i=0.585$ & 3744.14 & 3585.83 & 3331.8 & 3258.17 & 2989.42 \\
\hline$i=0.9$ & 11390.72 & 11040.24 & 10051.38 & 9438.03 & 8661.96 \\
\hline \hline Stroke/mm & 36 & 42 & 48 & 54 & 30 \\
\hline$i=0.585$ & 2521.86 & 2326.74 & 2080.076 & 1711.92 & 1675.11 \\
\hline$i=0.9$ & 7109.82 & 5407.47 & 5031.95 & 4155.74 & 4093.15 \\
\hline
\end{tabular}

Converting the data from the above table to the lifting force coefficient, in order to compare with the experimental data given by the field test personnel.

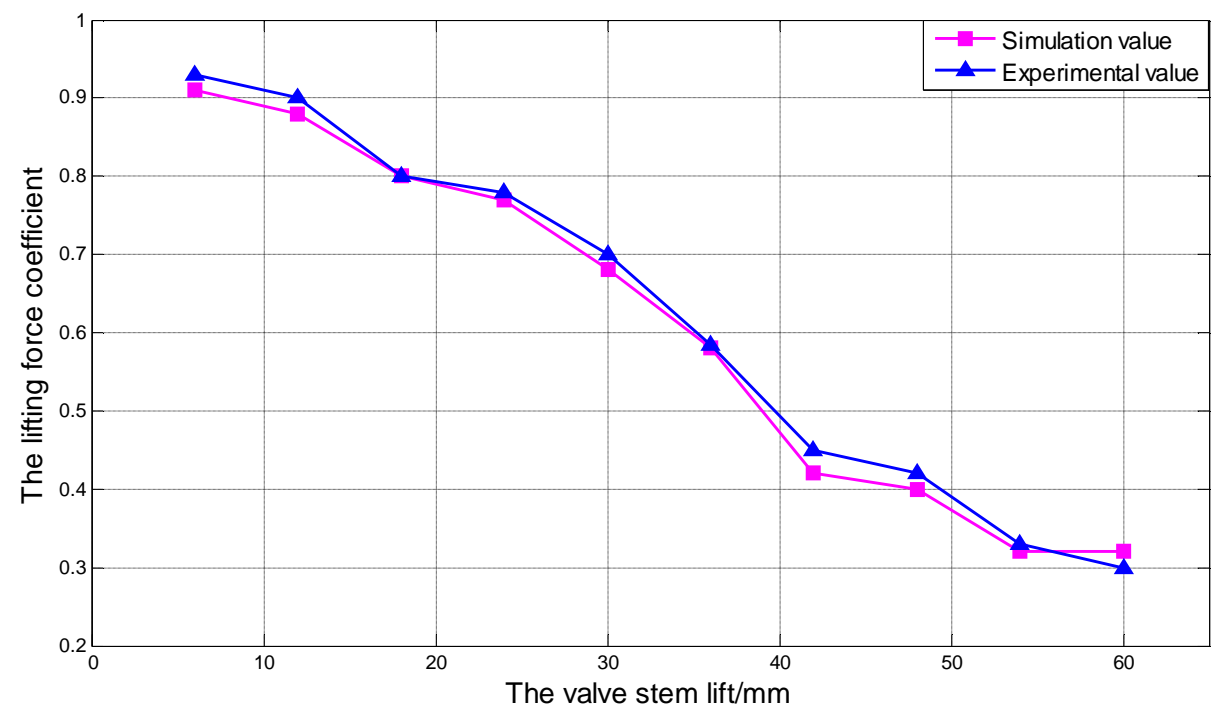

(a) $i=0.585$

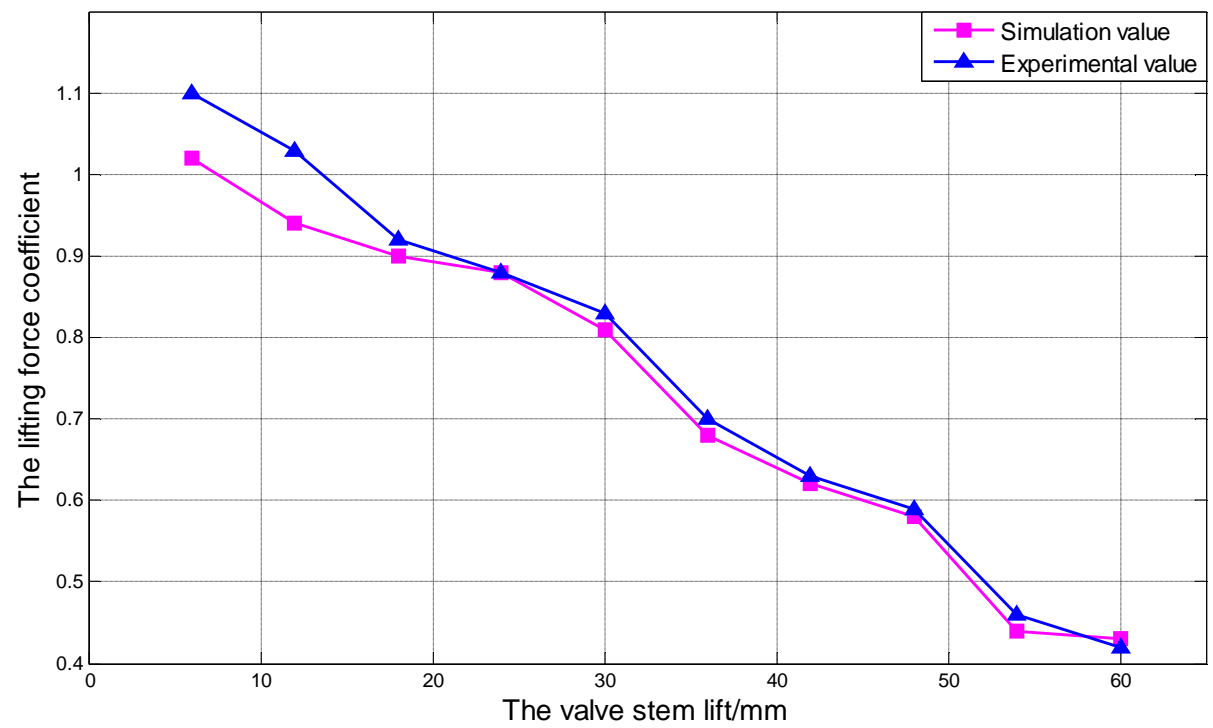

(b) $i=0.9$

Fig. 5 The stem lifting force curve of under different conditions

Figure 5 shows that with the increasing of the opening degree of the regulating valve, the lifting force decreases, and the variation of the approximation is linear. The simulation results are in agreement with the experimental values, and the error is small. It can also prove the rationality of the numerical simulation of the flow field. 


\section{Conclusions}

Through the above analysis, can be obtained in the process of regulating valve opening, and the opening of the valve opening of the relationship between the lifting force, specific as follows:

(1) In the pre start process, with the increase of the adjustment valve pre start stroke, the valve stem lifting force gradually decreases, when the pre start stroke to reach $3 \mathrm{~mm}$, the lifting force basically no change;

(2) In the process of regulating valve, the valve stem lifting force with the increase of the valve opening and decreases, and the change trend is similar to linear.

\section{References}

[1] Yang R. Predicting hydraulic valve flow forces using CFD. ASME 2004 International Mechanical Engineering Congress and Exposition. American Society of Mechanical Engineers, 2004:55-61P

[2] Ferziger J H, Perić M. Computational methods for fluid dynamics[M]. Berlin:Springer, 1996:147-149P

[3] An Y J, Kim B J, Shin B R. Numerical analysis of 3-D flow through LNG marine control valves for their advanced design[J]. Journal of mechanical science and technology, 2008, 22(10):1998-2005P

[4] Cho T D, Yang S M, Lee H Y, et al. A study on the force balance of an unbalanced globe valve. Journal of mechanical science and technology, 2007, 21(5):814-820P 